\title{
O modelo dos múltiplos fluxos de Kingdon na análise de políticas de saúde: aplicabilidades, contribuições e limites
}

\author{
Kingdon's multiple stream model used to analyze health \\ policies: applicability, contribution and limits
}

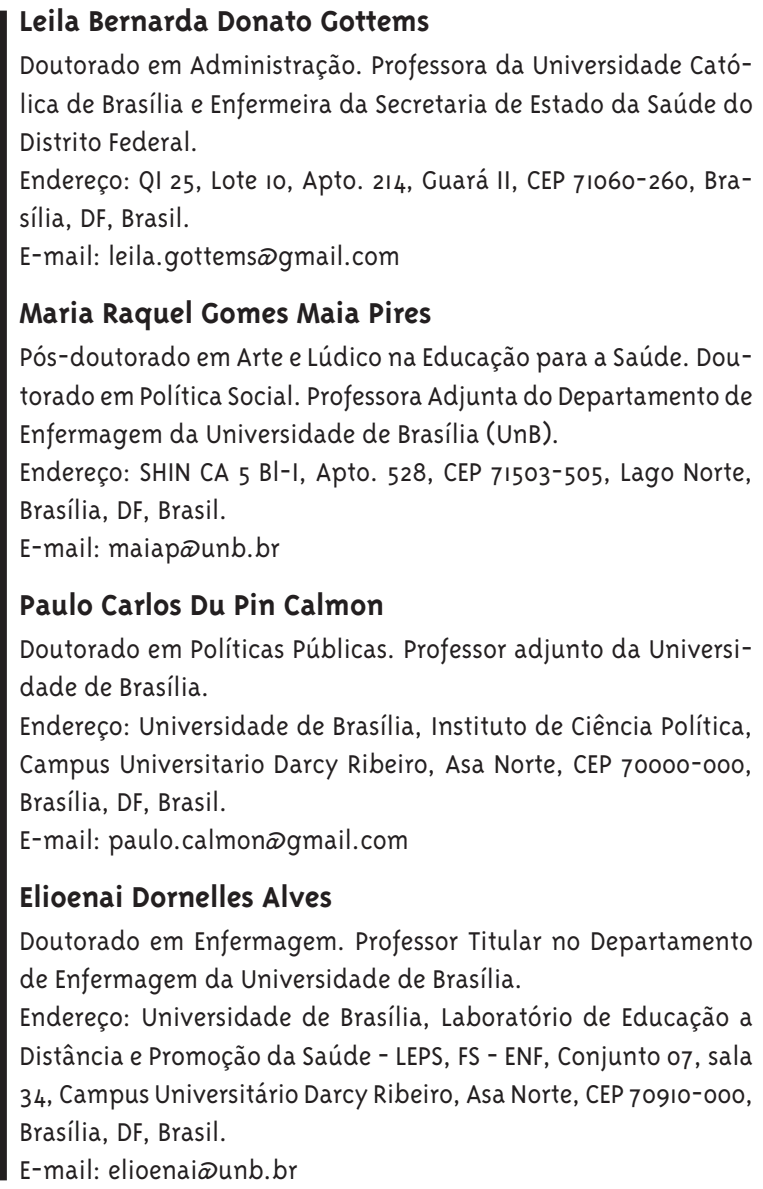

Pós-doutorado em Arte e Lúdico na Educação para a Saúde. Doutorado em Política Social. Professora Adjunta do Departamento de Enfermagem da Universidade de Brasília (UnB).

Endereço: SHIN CA 5 Bl-I, Apto. 528, CEP 71503-505, Lago Norte, Brasília, DF, Brasil.

E-mail: maiapœunb.br

\section{Paulo Carlos Du Pin Calmon}

Doutorado em Políticas Públicas. Professor adjunto da Universidade de Brasília.

Endereço: Universidade de Brasília, Instituto de Ciência Política, Campus Universitario Darcy Ribeiro, Asa Norte, CEP 70000-000, Brasília, DF, Brasil.

E-mail: paulo.calmonळgmail.com

\section{Elioenai Dornelles Alves}

Doutorado em Enfermagem. Professor Titular no Departamento de Enfermagem da Universidade de Brasília.

Endereço: Universidade de Brasília, Laboratório de Educação a Distância e Promoção da Saúde - LEPS, FS - ENF, Conjunto 07, sala 34, Campus Universitário Darcy Ribeiro, Asa Norte, CEP 70910-000, Brasília, DF, Brasil.

E-mail: elioenai®unb.br

\section{Resumo}

O presente trabalho teve como objetivos identificar as produções científicas sobre análise de políticas de saúde que utilizaram o modelo dos múltiplos fluxos de Kingdon e examinar as contribuições e os limites do modelo no estudo das políticas públicas de saúde, no contexto do SUS. Realizou-se uma revisão integrativa de publicações disponíveis nas bases de dados ProQuest, Portal de Periódicos e Banco de Teses e Dissertações da Capes e Scientific Electronic Library Online, com uso das palavras-chave Kingdon Model, multiple streams model (modelo dos múltiplos fluxos) e políticas públicas de saúde. Os critérios de inclusão foram: estudos completos em português e inglês, disponíveis on-line nas bases de dados; e análises de políticas públicas de saúde com uso do modelo Kingdon em experiências nacionais ou internacionais. Foram excluídos estudos de revisão e relatos de experiência. Foram levantados 4 teses de doutorado, 6 artigos e 2 dissertações de mestrado, publicados entre 1994 a 2009 . 0 modelo de Kingdon mostrou-se aplicável a análises de políticas de saúde em diferentes sistemas políticos nacional e internacional, em períodos prolongados de tempo e na atuação dos governos. As contribuições desse modelo de análise para o SUS incluem: a) a incorporação da ambiguidade nas decisões; b) a valorização da consistência das ideias contidas nas propostas; c) a análise das diferentes interpretações sobre os problemas complexos da saúde brasileira pelos tomadores de decisão. Dentre os limites, aponte-se a ênfase na atuação dos atores em detrimento do marco institucional que delimita suas decisões.

Palavras-chave: Políticas públicas; Políticas de saúde; Sistema de saúde. 


\section{Abstract}

This study aimed to identify the scientific production on health policy analysis that use Kingdon's model of multiple streams, and to examine the model's contributions and limits in the study of public health policies, in the SUS context. An integrative review of publications using the keywords Model Kingdon, multiple streams model "modelo de múltiplos fluxos" and health public policy was made at the following databases: ProQuest, Portal de Periódicos and Banco de Teses e Dissertações (from CAPES) and Scientific Electronic Library Online. Inclusion criteria were: complete studies in Portuguese and English, available online in the databases; analysis of public health policies using the Kingdon model in national or international experiences. Review studies and experience reports were excluded. We identified 4 doctoral thesis, 6 articles and 2 master dissertations published from 1994 to 2009. The Kingdon's model proved to be applicable for analysis of health policies in different national and international political systems, in long periods of time and in the practice of governments. The contributions of this model for SUS analysis include: a - the incorporation of ambiguity in the decisions; $b$-appreciation of the consistency of the ideas contained in the proposals; $\mathrm{C}$ - an analysis of different interpretations of decision makers about the complex problems of Brazilian health. Concerning its limits, we point out its emphasis in the action of actors at the expense of the institutional framework that delineates their decisions.

Keywords: Public Policy; Health Policy; Health System.

\section{Introdução}

As políticas de saúde no contexto do Sistema Único de Saúde (SUS) se desenvolvem em cenários permeados pela ambiguidade, pela incerteza e pelo conflito entre valores e interesses de grupos na escolha pública (Baggott, 2002; Walt e Gilson, 1994). As correlações de forças estão presentes na maioria das decisões, em organizações de saúde complexas, nas quais interagem múltiplos atores, com preferências e identidades heterogêneas (Arretche, 2004; Cortês, 2009; Viana e Baptista, 2008). Em vista disso, e não por acaso, a opção por processos pactuados em arenas políticas do SUS, ambientados em fóruns deliberativos e consultivos específicos, assumem crescente protagonismo e relevância no processo de democratização das políticas públicas de saúde no Brasil (Guerreiro e Branco, 2011).

A compreensão desses processos requer análises que tratem da dimensão política das políticas de saúde. Ou seja, contempla o exercício do poder e a racionalidade contida nas propostas de organização, de reorganização e de escolha de prioridades (Cohn, 2008). Esse esforço pode ser facilitado pelo diálogo entre diferentes campos do conhecimento, em especial entre a saúde coletiva e as políticas públicas.

A análise de políticas públicas implica o estudo do governo em ação e/ou do conjunto de ações que este realiza, ou não, para alcançar objetivos em relação a um problema ou conflito social. Exige modelos teóricos que possibilitem a compreensão da realidade, os sentidos e os significados dos processos envolvidos nas operações. Na maioria das vezes, tais análises são orientadas por modelos que supõem que os tomadores de decisão possuem preferências e atuam em função delas. Essas tendências teóricas limitam as interpretações, as relações de causalidade e os objetivos a serem alcançados (Souza, 2003; Bonafont, 2004; Sabatier, 2007).

A teoria dos múltiplos fluxos se originou a partir de estudos sobre a formação da agenda pública, elaborada por Kingdon (2003) e Zahariadis (2007), com origem no modelo de decisão em organizações. Os autores preconizam a presença da ambiguidade na tomada de decisão em função de três fatores: (i) participação fluida pela grande rotatividade dos atores envolvidos nas arenas decisórias; (ii) preferências 
vulneráveis, vez que os tomadores de decisão não compreendem com clareza o impacto de suas decisões e, portanto, não conseguem se posicionar em relação às diferentes alternativas diante deles; (iii) tecnologias mal definidas, especialmente no setor público, marcado por crescente interdependência, disputas intra e intergovernamentais, conflitos jurisdicionais, contestações em relação à divisão de atribuições e de responsabilidades. Ambiguidade está relacionada à presença de ambivalência, ou seja, à presença simultânea de diferentes interpretações, muitas vezes conflitantes, sobre um mesmo fenômeno. Incerteza, por outro lado, decorre da ausência de informação (Kingdon, 2003; Zahariadis, 2007).

Kingdon (2003), seguindo Cohen, March e Olsen (1972), propõe que o processo de tomada de decisão nas políticas públicas poderia ser representado pela confluência de três grandes correntes dinâmicas: a corrente dos problemas (problems), a das propostas ou alternativas (policies) e a da política (politics). Assim, a agenda governamental segue um processo não intencional que se caracteriza por:1) surgimento ou reconhecimento de um problema pela sociedade em geral; 2) existência de ideias e alternativas para conceituá-los - originadas de especialistas, investigadores, políticos e atores sociais, dentre outros; 3) contexto político, administrativo e legislativo favorável ao desenvolvimento da ação (Figura 1) (Kingdon, 2003; Bonafont, 2004; Ferrarezi, 2007; Zahariadis, 2007; Capella, 2007).

O fluxo dos problemas do modelo Kingdon analisa de que forma as questões são reconhecidas e os motivos que as levam a compor a agenda do governo. A atenção governamental se daria em função de três acontecimentos (Kingdon, 2003): 1) indicadores que apontam e mensuram a magnitude de uma situação; 2) a ocorrência de eventos, crises, desastres ou uma experiência pessoal; e 3) o feedback oriundo do monitoramento sobre orçamento, custos e gastos. A resposta dos formuladores, por sua vez, depende da forma como estes percebem e interpretam a realidade (Capella, 2007). Ressalta-se a relevância dos indicadores sociais e epidemiológicos, expressivos no setor saúde, como importantes para desvelar a magnitude dos problemas e influenciar a formação da agenda pública do setor.

\section{Figura I - 0 modelo dos múltiplos fluxos de Kingdon}

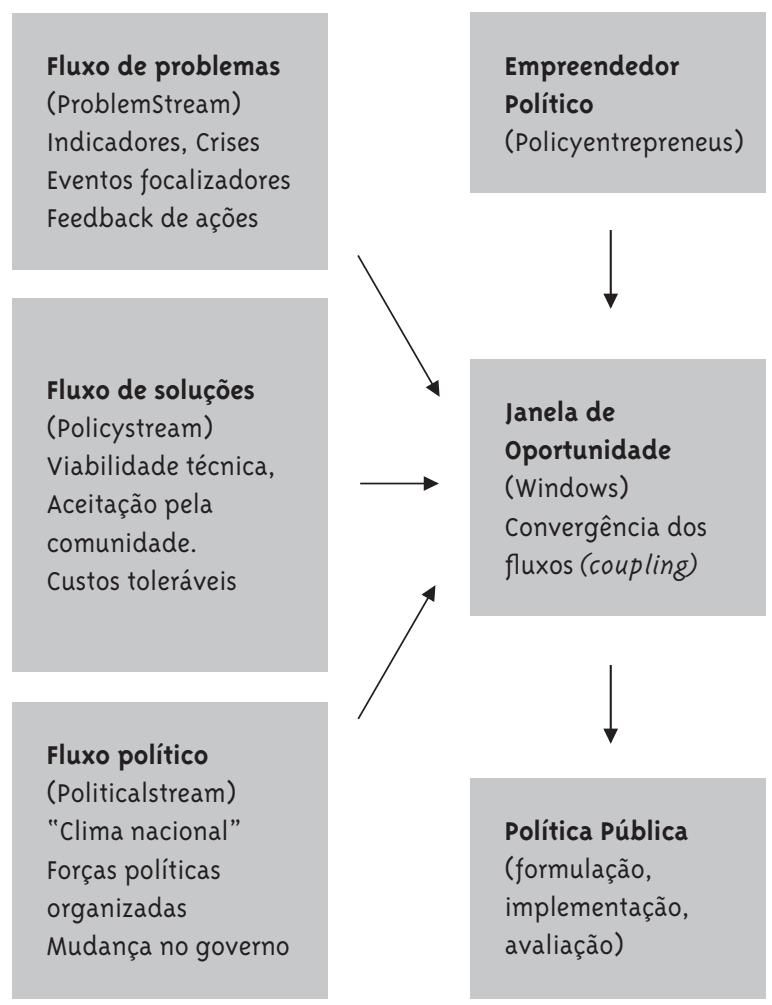

Fonte: adaptado de Capella, 2007: pág. 98 e Zahariadis, 2007: pág. 71.

O fluxo das alternativas inclui o conjunto de ideias e propostas em disputa para ganhar aceitação na rede de políticas. Nesse processo nem todas as ideias recebem o mesmo tratamento - algumas permanecem intocadas, outras são combinadas a novas propostas e/ou desaparecem. Entre as alternativas que originam as políticas públicas é possível identificar alguns padrões, tais como: a confiabilidade técnica; a aceitabilidade e compatibilidade entre os valores vigentes na sociedade; e a capacidade de antecipar contingenciamentos futuros, como os orçamentários (Kingdon, 2003).

No fluxo político do modelo dos múltiplos fluxos três elementos são considerados: o clima nacional (national mood), as forças políticas organizadas e as mudanças no governo (Kingdon, 2003; Zahariadis, 2007). O clima nacional se caracteriza pelo compartilhamento das questões relevantes na sociedade, configurando um ambiente favorável para a formação da agenda política. As forças políticas organizadas 
referem-se às pressões exercidas por grupos, levando a consensos ou conflitos na arena política. Por fim, as mudanças no governo influenciam a agenda tanto de pessoas em posições estratégicas como na composição do legislativo. Chama-se especial atenção para o início de governos, considerado o momento mais propício à entrada de demandas que permaneceram por um longo tempo sem resposta (Kingdon, 2003).

Os atores de uma política pública se dividem entre os governamentais - o staff do poder executivo e os servidores de carreira - e os não governamentais - compostos pelos grupos de interesse e pelos grupos acadêmicos. Aqueles que exercem um papel fundamental na integração dos fluxos são os empreendedores de políticas públicas, que detêm qualidades básicas, tais como: o apelo para se fazer ouvir, articulações políticas, habilidades para negociar e persistência. A integração dos três fluxos - problemas, alternativas e política - é denominada como a abertura de uma janela de políticas públicas, que se constitui na oportunidade para que os empreendedores possam apresentar suas propostas. $\mathrm{O}$ fechamento das janelas pode ocorrer após a tomada de alguma decisão, pelo fracasso em buscar uma solução, pela perda de visibilidade da questão, por mudanças do pessoal envolvido no tema ou simplesmente por não haver uma alternativa de ação disponível (Kingdon, 2003; Bonafont, 2004; Ferrarezi, 2007; Zahariadis, 2007; Capella, 2007).

Diante desse contexto, indaga-se neste artigo quais as aplicabilidades, os potenciais e os limites do modelo dos múltiplos fluxos de Kingdon (2003) na análise da dimensão política das políticas de saúde no contexto do SUS. Têm-se como objetivos: 1) identificar as produções científicas sobre análise de políticas de saúde que utilizaram o modelo dos múltiplos fluxos de Kingdon; e 2) examinar as contribuições e os limites do modelo no estudo das políticas públicas de saúde, no contexto do SUS.

\section{Metodologia}

Trata-se de uma revisão integrativa de literatura, método específico que permite produzir um mapeamento de determinado fenômeno em particular, traçando-lhe uma análise sobre o conhecimento já produzido sobre o tema (Mendes e col., 2008). Realizaram-se as fases previstas na revisão integrativa, ou seja, identificação do tema, busca na literatura, categorização dos estudos, avaliação e interpretação dos resultados, síntese do conhecimento explicitado nos estudos analisados. 0 artigo integra tese de doutorado que analisou a política de atenção básica no Distrito Federal com uso no modelo dos múltiplos fluxos (Göttems, 2010).

A revisão abrangeu teses, dissertações e artigos nacionais e internacionais disponíveis nas bases de dados do ProQuest Dissertations \& Theses Database (http://www.proquest.com/en-US/catalogs/databases/detail/pqdt.shtml), do Portal de Periódicos e Banco de Teses e Dissertações da Capes (www. periodicos.capes.gov.br), bem como da Scientific Electronic Library Online (http://www.scielo.br). Foram utilizados como expressões-chave e descritores os termos "Kingdon Model”, multiple streams model (modelo dos múltiplos fluxos) e políticas públicas de saúde. Em uma primeira busca foram levantados 4 teses de doutorado, 6 artigos e 2 dissertações de mestrado, publicados entre 1994 a 2009. Os critérios de inclusão foram: estudos completos em português e inglês, disponíveis on-line nas bases de dados; e análises de políticas públicas de saúde com uso do modelo Kingdon em experiências nacionais ou internacionais. Após a leitura das publicações, excluiu-se uma tese de doutorado e um dos artigos por não abordarem o setor saúde. Foram examinados, nas investigações selecionadas, a aplicação do modelo na área de saúde, os potenciais e os limites do marco teórico. Excluiu-se das análises o detalhamento de informações metodológicas e de resultados, salvo nos casos em que foram necessárias para compreender a aplicação do modelo dos múltiplos fluxos de Kingdon (2003).

\section{Resultados}

As publicações que utilizaram o modelo Kingdon (2003) e que foram selecionadas para este estudo mediante os critérios definidos apresentam diversidade de objetos, tempos e cenários, conforme verifica-se no Quadro 1. A seguir, discorre-se sobre cada uma das investigações analisadas. 


\section{Quadro I - Síntese das produções teóricas analisadas}

\begin{tabular}{|c|c|}
\hline Referências & Objetos \\
\hline McEldowney, 1994 & $\begin{array}{l}\text { Examinou as grandes deliberações públicas que ocorreram durante um período de um século nos sistemas } \\
\text { de saúde dos EUA e da Grã-Bretanha. }\end{array}$ \\
\hline Petchey e col., 2008 & $\begin{array}{l}\text { Estudaram a relação entre política central e implementação local no contexto da criação de fundos } \\
\text { lotéricos para desenvolver sistemas comunitários de cuidados com câncer no Reino Unido. }\end{array}$ \\
\hline Exworthye col., 2002 & $\begin{array}{l}\text { Buscaram explicar como as questões referentes às desigualdades em saúde entraram na agenda política } \\
\text { nacional e local e criaram oportunidades para a efetiva implementação de ações durante o governo } \\
\text { trabalhista no Reino Unido. }\end{array}$ \\
\hline Mannheimere col.,2007 & $\begin{array}{l}\text { Analisaram a formação da agenda, a formulação, início e implementação da política pública intersetorial } \\
\text { de saúde, seu impacto nos níveis local e nacional, no município de Estocolmo na Suécia. }\end{array}$ \\
\hline L H Ho, 2002 & $\begin{array}{l}\text { Estudou o processo de agenda setting para a regulação do trabalho dos médicos tradicionais chineses em } \\
\text { Hong Kong. }\end{array}$ \\
\hline Onieal, 2005 & $\begin{array}{l}\text { Examinou as perspectivas governamentais, comunitárias e dos provedores de serviços (profissionais de } \\
\text { saúde) a respeito do fechamento do Waltham Hospital em Massachusetts em } 2003 .\end{array}$ \\
\hline Mitchell, 1998 & $\begin{array}{l}\text { Analisou o processo de decisão política de Rudolf Giulianis que o levou à privatização dos hospitais } \\
\text { municipais de Nova York durante sua gestão como prefeito daquela cidade. }\end{array}$ \\
\hline Monteiro,2006 & $\begin{array}{l}\text { Analisou os processos de formulação e implementação do Programa Nacional de Doenças Sexualmente } \\
\text { Transmissíveis e AIDS, entre os anos } 1980 \text { e } 2006 \text {, no Brasil. }\end{array}$ \\
\hline Machado, 2006 & $\begin{array}{l}\text { Analisou as políticas priorizadas pelo Ministério da Saúde brasileiro entre } 1990 \text { e } 2002 \text {, considerando a } \\
\text { relação entre as agendas de reforma que repercutiram sobre a saúde e a inserção da política setorial no } \\
\text { projeto governamental. Usa o conceito de Kingdon sobre agenda do governo. }\end{array}$ \\
\hline Czermainski, 2009 & $\begin{array}{l}\text { Analisou os antecedentes da formação da agenda das políticas públicas brasileiras de assistência } \\
\text { farmacêutica e o processo de formação da agenda e da política de plantas medicinais e fitoterápicos. }\end{array}$ \\
\hline
\end{tabular}

O estudo que compara as grandes decisões sobre os sistemas de saúde dos Estados Unidos da América (EUA) e Grã-Bretanha (McEldowney, 1994) investigou a oferta de serviços de saúde à população, incluindo os processos e as forças políticas que influenciaram as políticas de saúde. Na trajetória do sistema de saúde dos EUA, aponta-se a convergência de dois temas: negócios e governo. O governo enfrentava limites para arcar com as despesas correntes com os serviços de saúde e não visualizava um fim nos crescentes custos com a prestação dos cuidados. Essas ideias promoveram uma quarta tentativa de se estabelecer um sistema nacional de saúde no governo de Bill Clinton. Contudo, a convergência dos três fluxos políticos no contexto norte-americano foi inviabilizada pelo conflito de interesses e pela desinformação entre os atores (McEldowney, 1994). Além disso, a força das ideias originais se perdeu nos numerosos planos de reforma do setor saúde e nos conflitos partidários, impedindo a completa abertura de uma janela de oportunidade política.
A reforma do setor saúde britânico teve trajetória curta e consolidada no governo de Margaret Thatcher. A proposta teve origem no alto escalão governamental e foi rapidamente objeto de uma campanha política que permitiu pouco espaço para dissenso. A janela de oportunidade para essa proposta já estava parcialmente aberta pelo desejo de livre mercado existente no âmbito dos prestadores de serviços de saúde, que desenvolviam uma administração competitiva. A reforma foi marcada pela concorrência na oferta dos serviços, ainda que com controle do legislativo. Essa foi uma medida que marcou uma ruptura com o passado e conduziu a Grã-Bretanha a mudanças nos processos políticos (McEldowney, 1994).

Na pesquisa sobre a criação de fundos lotéricos para desenvolver projetos comunitários de assistência ao câncer no Reino Unido, realizado por Petchey e colaboradores (2008), após a era Thatcher, examinaram-se as relações entre o governo central, as propostas e os aspectos econômicos da implantação 
local. No que se refere ao impacto do financiamento nas ações de saúde voltadas a pessoas com câncer, apontaram-se diversificação na oferta de serviços oncológicos e priorização das necessidades da demanda desassistida. Os autores creditaram o êxito da política aos empreendedores locais pela capacidade de agregar os fluxos de problemas ao ambiente político e às soluções (Petchey e col., 2008).

A análise das desigualdades sociais na saúde durante o governo trabalhista no Reino Unido, a partir de 1997, aprofundou o conhecimento sobre a atuação dos empreendedores políticos e sobre a abertura de janelas de oportunidade, em diferentes níveis governamentais (Exworthy e col., 2002). Nesse estudo, identificou-se que a atuação dos empreendedores locais foi influenciada pela ausência de articulação governamental no nível nacional, assim como pelas constantes mudanças nas prioridades políticas de curto prazo. No que se refere à dimensão vertical das políticas, analisaram-se as relações entre os níveis de governo, especialmente no tocante à possibilidade de reinterpretação local das diretrizes nacionais e à capacidade de acompanhamento do nível central. A pesquisa sinalizou que, na relação entre diferentes níveis de governos, as prioridades da agenda nacional competem significativamente com as prioridades da agenda local (Exworthy e col., 2002).

O processo de formação da agenda, a formulação, o início e a implantação da política pública foram investigados na Suécia norteados pela questão: "Como uma janela de oportunidade pode ser aberta para uma política pública e intersetorial de saúde a despeito da baixa prioridade política dada a esta questão?" (Mannheimer e col., 2007). Os resultados demonstraram que, naquele momento, havia uma forte relação entre a política de saúde e o contexto internacional; que o processo de mudança fora mais tecnicamente orientado, no nível nacional, e politicamente, no nível local; que os interesses na proposta evoluíram ao longo de uma década, para finalmente serem aprovados. Mannheimer e colaboradores (2007) usaram conceitos de high politics referindo-se a questões politicamente priorizadas; definiram como low politics as questões para as quais se confere pouco peso na coordenação global das políticas; e fortaleceram o argumento de King- don de que, embora a saúde figure como universalmente importante no setor público, raramente aparece entre as questões da alta ou macropolítica.

A regulação do trabalho dos médicos tradicionais chineses em Hong Kong foi analisada por L H Ho (2002) sob as questões: "Quais foram os catalisadores da mudança? Por que aconteceu naquele momento em particular? Quais foram os envolvidos? Como as questões ascenderam para a agenda do governo e então para a agenda de decisões?" A pesquisa observou que, no fluxo político, a atitude dos governos, dos médicos e do público foi fator-chave. No fluxo de problemas, identificou-se a ausência de regulação das práticas médicas, predominantes por mais de 150 anos. No fluxo das soluções, os autores identificaram três propostas: o estatuto regulatório para os médicos tradicionais chineses; a exigência de registro dos profissionais existentes; o controle do uso de medicamentos. A janela política foi possibilitada pelo enfraquecimento do governo na formulação de políticas, que permitiu que a questão da regulação médica entrasse na agenda pública (L H Ho, 2002).

O fechamento do Waltham Hospital em Massachusetts, em 2003, foi examinado por Oniel (2005) sob duas questões: 1) Quais foram as perspectivas governamentais, comunitárias e dos prestadores de serviços de saúde sobre o fechamento do hospital? e 2) Qual o impacto do fechamento do hospital no acesso aos serviços de saúde? 0 modelo de Kingdon foi aplicado para identificar os informantes-chave. 0 processo político foi analisado por meio do modelo de Stone (1997) apud Oniel (2005), sob a argumentação de que este amplia o modelo dos múltiplos fluxos, provendo-lhe meios para discutir as metas e os valores da política.

A pesquisa sobre o processo de decisão política de Rudolf Giulianis (Mitchell, 1998), que levou à privatização dos hospitais municipais de Nova York, durante sua gestão como prefeito, analisou os fatores que influenciaram essa medida e as forças políticas que interferiram no curso das ações. Os achados explicitaram a confluência entre o contexto mercadológico nos Estados Unidos, cujas raízes foram remetidas a Osborne e Gaebler (1992), associada à ideia de governança que concede ao governo o papel de coordenador das metas coletivas e ao mercado, o desafio de alcançá-las. A abertura da 
janela política para a privatização foi atrelada aos históricos problemas da rede hospitalar de Nova York - déficit financeiro, baixa qualidade nos serviços, malversação de recursos públicos, descuido no pagamento dos impostos - associados à atuação de empreendedor político importante, o próprio prefeito recém-eleito (Mitchell, 1998).

No contexto brasileiro, Monteiro (2006) analisou os processos de formulação e implantação do Programa Nacional de Doenças Sexualmente Transmissíveis (DST) e Aids, entre os anos 1980 e 2006. A autora aplicou o modelo conceitual de Kingdon em relação aos processos pré-decisionais que antecedem à implementação de uma política social: a construção da agenda governamental e a especificação das alternativas a serem escolhidas, bem como a correlação de forças e a articulação entre os atores do Estado e da sociedade civil. Os resultados demonstraram a complexidade dos processos de formulação de políticas de saúde no Brasil, assim como apresentaram a dinâmica das inter-relações entre os atores e o papel dos movimentos sociais como relevantes.

0 conceito de agenda - tema que mobiliza fortemente a atenção dos atores governamentais e não governamentais (Kingdon, 2003) - foi aplicado por Machado (2006) no mapeamento das prioridades do Ministério da Saúde (MS), entre os anos 1990 e 2002. Os resultados indicaram que a descentralização do SUS, a Estratégia Saúde da Família (ESF) e a política de combate à aids foram as prioridades daquele período. Indicaram também que a agenda nacional foi influenciada pelas diferentes reformas, pela constituição de uma base social de apoio, pela formação de grupos técnicos no MS e pelo caráter atrativo dos programas de governo.

A formação da Politica Nacional de Plantas Medicinais e Fitoterápicos (PNPMF) foi analisada por Czermainski (2009) para elucidar "em que medida as questões que demandaram a política nacional de plantas medicinais e fitoterápicos podem se refletir na sua implementação e resultados" (p. 6). A autora reconstituiu a formação da politica de assistência farmacêutica como evento prévio à PNPMF, desenhou o processo de convergência dos fluxos e identificou a atuação dos atores e empreendedores na abertura da janela de oportunidade para a PNPMF.
Os resultados, ainda que não conclusivos, possibilitaram a elaboração de recomendações para a gestão da saúde no Estado do Rio Grande do Sul, bem como apontaram questões a serem aprofundadas em outros estudos.

\section{Discussão}

No que se refere à aplicação do modelo de Kingdon na análise de políticas de saúde, observou-se maior frequência na literatura internacional (Mceldowney, 1994; Petchey e col., 2008; Exworthy e col., 2002; Mannheimer e col., 2007; L h ho, 2002; Mitchell, 1998; Oniel, 2005) do que na nacional (Monteiro, 2006; Machado, 2006; Czermainski, 2009). Em relação ao objeto estudado, identificaram-se diversidades que incluem: as grandes deliberações públicas sobre os sistemas de saúde (Mceldowney, 1994); a dimensão vertical das políticas de saúde; as relações entre níveis de governo e destes com a sociedade civil (Petchey e col., 2008; Exworthy e col., 2002; Oniel, 2005); a formação da agenda, a formulação, o início e a implantação da política (Exworthy e col., 2002; Monteiro, 2006; Machado, 2006; Czermainski, 2009). 0 modelo também é aplicado para estudar agendas internas do setor saúde, como a regulação do trabalho dos médicos tradicionais chineses em Hong Kong (L h ho, 2002) e as agendas do Estado brasileiro na política de saúde (Monteiro, 2006; Machado, 2006; Czermainski, 2009).

Em todos os estudos, o governo e suas decisões figuraram como questões centrais, excetuando-se aqueles que se debruçaram sobre a privatização (Mitchell, 1998; Oniel, 2005). Em relação à dimensão temporal das pesquisas, observaram-se análises de processos políticos que perduraram um século (Mceldowney, 1994), décadas (Mitchell, 1998; Oniel, 2005; Monteiro, 2006; Czermainski, 2009), e outros cuja variável tempo não figurou como fundamental na compreensão das mudanças nas políticas públicas (Petchey e col., 2008; Exworthy e col., 2002; Mannheimer e col., 2007; L h ho, 2002).

O modelo de Kingdon mostrou-se aplicável em diferentes sistemas políticos, para análises de períodos prolongados de tempo, exame de interações contemporâneas entre nações, delimitação das influências político-ideológicas na formulação das po- 
líticas públicas e do papel das novas administrações na abertura de uma janela política. Ao ser aplicado, possibilitou explicitar a importância dos indicadores econômicos e sociais nas priorizações das ações de governos. Em linhas gerais, as contribuições do modelo dos múltiplos fluxos para a análise de políticas públicas podem ser divididas em quatro eixos: 1) no estudo da macropolítica e sua influência nas políticas setoriais; 2) na análise das influências das relações intergovernamentais e dos governos com a sociedade civil na formação da agenda; 3) no exame da atuação dos atores e empreendedores na implantação da política; 4) no estudo dos processos decisórios dos governos locais e nacionais.

A partir dos estudos analisados, verificou-se que tanto a amplitude das variáveis como a incorporação da ambiguidade nas decisões sobre políticas públicas, bem como a valorização da consistência das ideias contidas nas propostas, constituem-se grandes contribuições do modelo de Kingdon (2003) para o estudo das políticas de saúde no Brasil. 0 modelo configura-se estratégia teórica e metodológica relevante para desenhos de pesquisas avaliativas. Especialmente nos estudo de natureza política, o modelo dos múltiplos fluxos parece apresentar maior coerência, tendo em vista que considera as ambivalências e incertezas, bem como a influência de grupos de interesses nas escolhas políticas (Czermainski, 2009; Baggott, 2002). Além disso, as variáveis amplas - que incluem os problemas, as alternativas e o meio político - permitem lidar com as diferentes interpretações sobre os problemas e como estes captam a atenção dos atores em diferentes níveis de governo e fóruns, desvelando a subjetividade dos tomadores de decisão.

Porém, os principais limites do modelo dos múltiplos fluxos, de forma geral, referem-se à baixa capacidade preditiva, à subespecificação das forças causais, à concentração nos fatores situacionais e temporais, sua ênfase descritiva e as dúvidas sobre o caráter incremental na especificação de alternativas (Ferrarezi, 2007). Outras limitações dizem respeito ao enfoque nos atores e nas relações interpessoais, deixando à margem o marco institucional em que os mesmos levam suas ideias a cabo (Bonafont, 2004). Nos estudos analisados percebeu-se que a combinação do modelo dos múltiplos fluxos com outros constructos teóricos - que possibilitam abordar as dimensões tempo e história - constituiu-se uma estratégia metodológica adequada para diminuir os limites do modelo, aumentando-lhe seu potencial analítico (Mceldowney, 1994; L h ho, 2002; Monteiro, 2006; Machado, 2006).

\section{Conclusão}

As produções sobre análise de políticas de saúde que utilizaram o modelo dos múltiplos fluxos concentram-se em estudos internacionais, com foco na atuação dos governos, com objetos, temporalidades e contextos políticos diversos. As principais contribuições e potencialidades do modelo para a análise das políticas de saúde no âmbito do SUS são: a) a incorporação da ambiguidade nas decisões; b) a valorização da consistência das ideias contidas nas propostas; c) a análise das diferentes interpretações sobre os problemas complexos da saúde brasileira pelos tomadores de decisão; d) a influência da macropolítica, das relações intergovernamentais e da sociedade civil na formação da agenda pública; e) o exame acurado da atuação dos atores e dos empreendedores nos processos decisórios locais, nacionais e nas arenas políticas.

Os limites do modelo na análise das políticas públicas de saúde do Brasil constituem-se em: a) baixa capacidade preditiva; b) ênfase descritiva dos aspectos situacionais e temporais das mudanças políticas; c) foco na atuação dos atores e suas relações interpessoais em detrimento do marco institucional que delimita suas decisões. Para aumentar o potencial analítico do modelo recomenda-se a combinação deste com outros constructos teóricos que possibilitam abordar o peso das instituições, do tempo e da historicidade das mudanças políticas.

\section{Referências}

ARRETCHE, M. Federalismo e políticas sociais no Brasil: problemas de coordenação e autonomia. São Paulo em Perspectiva, São Paulo, v. 18, n. 2, p. 17-26, 2004.

BAGGOTT, R. Public health: political issues and policy dilemmas. Consumer Policy Review, London, v. 12, n. 1, p. 2-8, 2002. 
BONAFONT, L. C. Redes de políticas públicas. Madrid: Centro de Investigaciones Sociológicas, 2004 .

CAPELLA, A. C. N. Perspectivas teóricas sobre o processo de formulação de políticas públicas. In: HOCHMAN, G. et al. (Org.). Políticas públicas no Brasil. Rio de Janeiro: Fiocruz, 2007. p. 87-121.

COHEN, M. D.; MARCH, J. G.; OLSEN, J. P. A garbage can model of organizational choice. Administrative Science Quarterly, Ithaca, v. 17, n. 1, p. 1-25, 1972.

COHN, A. O estudo das políticas de saúde: implicações e fatos. In: CAMPOS, G. W. S. et al. (Org.). Tratado de saúde coletiva. São Paulo: Hucitec; Rio de Janeiro: Fiocruz, 2008. p. 219-246.

CÔRTES, S. V. Sistema Único de Saúde: espaços decisórios e a arena política de saúde. Caderno de Saúde Pública, Rio de Janeiro, v. 25, n. 7, p. 1626 1633, 2009.

CZERMAINSKI, S. B. C. A Politica Nacional de Plantas Medicinais e Fitoterápicos: um estudo a partir da análise de politicas públicas. 2009. Dissertação (Mestrado Profissional em Gestão da Assistência Farmacêutica) - Faculdade de Farmácia da Universidade Federal do Rio Grande do Sul, Porto Alegre, 2009.

EXWORTHY, M.; BLANE, D.; MARMOT, M. Tackling health inequalities in the United Kingdom: the progress and Pitfalls of Policy. HSR: Health Services Research. London, v. 38, n. 6, p. 1905-1922, 2002. Part II.

FERRAREZI, E. R. A reforma do marco legal do terceiro setor no Brasil: a criação da lei das OSCIP (Lei 9.790/99). 2007. Tese (Doutorado em Sociologia) - Departamento de Sociologia da Universidade de Brasília, Brasília 2007.

GÖTTEMS, L. B. D. Análise da política de Atenção Primária à Saúde desenvolvida no Distrito Federal: a articulação entre o contexto político, os problemas, as alternativas e os atores na formação da política de saúde (1979 a 2009). 2010. Tese (Doutorado em Administração) - Faculdade de Administração, Contabilidade e Economia da Universidade de Brasília, Brasília, 2010.
GUERREIRO, J. V.; BRANCO, M. A. F. Dos pactos políticos à política dos pactos na saúde. Ciência e Saúde Coletiva, Rio de Janeiro, v. 16, n. 3, p. 16891698, 2011.

KINGDON, J. W. Agendas, alternativas and public policies. 2. ed. Ann Arbor, : University of Michigan, 2003.

L. H. HO, P. Agenda-setting for the regulation of traditional Chinese medicine in Hong Kong. Asian Journal of Public Administration, Hong Kong, v. 24, n. 2, p. 257-285, 2002.

MACHADO, C. V. Prioridades de saúde no Brasil nos anos 1990: três políticas, muitas lições. Revista Panamericana de Salud Pública, Washington, DC, v. 20, n. 1, p. 521-532, 2006.

MANNHEIMER, L. N.; LEHTO, J.; ÖSTLIN, P. Window of opportunity for intersectoral health policy in Sweden-open, half-open or half-shut? Health Promotion International, Victoria, v. 22, n. 4, p. 307-315, 2007.

McELDOWNEY, R. P. A century of democratic deliberation over American and British national health care: extending the Kingdon model. 1994. Tese (Doctor of Philosophy) - Virginia Polytechnic Institute and State University, Blacksburg, 1994.

MENDES, K. D. S.; SILVEIRA, R. C. C. P.; GALVÃO, C. M. Revisão integrativa: método de pesquisa para a incorporação de evidências na saúde e enfermagem. Texto e Contexto Enfermagem, Florianópolis, v. 17, n. 4, p. 758-764, 2008.

MITCHELL, M. K. Privatizing New York city's public hospitals: the politics of policy making. 1998. Tese (Doctorate in Philosophy) - The City University of New York, New York, 1998.

MONTEIRO, A. L. O. M. A relação estado e sociedade civil no processo de formulação $e$ implementação de políticas públicas: análise do Programa Nacional de DST e AIDS (19802006). 2006. Dissertação (Mestrado em Política Social) - Faculdade de Economia, Administração, Contabilidade e Ciência da Informação e Documentação (FACE) da Universidade de Brasília, Brasília, DF, 2006. 
ONIEL, M. E. F. Hospital closures in Massachusetts: a case study of the Waltham Hospital closure. 2005. Dissertation (Nursing Program) - University of Massachusetts, Boston, 2005 .

OSBORNE, D.; GAEBLER, T. Reinventing government. Redding: Addison-Wesley, 1992.

PETCHEY, R.; WILLIAMS, J.; CARTER, Y. From street-leves bureaucrats to street-level policy entrepreneurs?: central policy and local action in lottery-funded community cancer care. Social Policy \& Administration, Wiley Drive, v. 42, n. 1, p. 59-76, 2008.

SABATIER, P. A. The need for better theories. In: SABATIER, P. A. (Ed.). Theories of the policy process. Boulder: Westview, 2007. p. 3-17.
SOUZA, C. Estado do campo da pesquisa em políticas públicas no Brasil. RBCS, São Paulo, v. 18, n. 51, p. 15-20, 2003.

VIANA, A. L. A.; BAPTISTA, T. W. F. Análise de políticas de saúde. In: GIOVANELLA, L. et al. (Org.). Políticas e sistemas de saúde no Brasil. Rio de Janeiro: Fiocruz, 2008. p. 65-105.

WALT, G.; GILSON, L. Reforming the health sector in developing countries: the central role of policy analysis. Health Policy and Planning, London, v. 9, n. 4, p. 353-370, 1994.

ZAHARIADIS, N. The multiple streams framework: structure, limitations, prospects. In: SABATIER, P. A. (Ed.). Theories of the policy process. Boulder: Westview, 2007. p. 65-92. 